\title{
EFECTO DE DOS SUSTRATOS Y DIFERENTES LÁMINAS DE RIEGO EN LA PROPAGACION DE ROMERO (Rosmarinus officinalis L.)
}

\section{EFFECT OF TWO SUBSTRATES AND DIFFERENT IRRIGATION LEVELS IN THE PROPAGATION OF ROSEMARY (Rosmarinus officinalis L.)}

\author{
Javier Giovanni Álvarez-Herrera' \\ Edinson Chacón Pardo ${ }^{2}$ \\ Santos Lusardo Rodríguez ${ }^{3}$
}

\begin{abstract}
RESUMEN
Existe poca información sobre láminas de riego y de sustratos adecuados para la propagación de romero bajo invernadero, que permitan al productor aprovechar la capacidad productiva total de la planta. Bajo condiciones de estrés, el romero limita la producción de biomasa seca y fresca. Se evaluó el efecto de dos sustratos y diferentes láminas de riego en la propagación del romero. Se realizó un diseño experimental completamente al azar con diez tratamientos, producto de la combinación de cinco láminas de riego (coeficientes multiplicadores de la evapotranspiración 0,$6 ; 0,8 ; 1 ; 1,2$ y 1,4) y dos sustratos: suelo negro (SN) y mezcla de suelo negro y cascarilla de arroz quemada (ME); cada tratamiento tuvo cuatro repeticiones para un total de 40 unidades experimentales (UE). El mejor coeficiente (lámina) para la propagación de esquejes de romero fue uno. El mejor sustrato para
\end{abstract}

${ }^{1}$ Profesor asistente. Grupo de Investigaciones Agrícolas, Facultad de Ciencias Agropecuarias, Universidad Pedagógica y Tecnológica de Colombia, Tunja. Av. Central del Norte. e-mail: jgalvarezh@gmail.com

2 Ingeniero Agrónomo. Grupo de Investigaciones Agrícolas, Facultad de Ciencias Agropecuarias, Universidad Pedagógica y Tecnológica de Colombia. Tunja. Av. Central del Norte. e-mail: ing.edinsonchacon@hotmail.com

3 Ingeniero Agrónomo. Grupo de Investigaciones Agrícolas, Facultad de Ciencias Agropecuarias, Universidad Pedagógica y Tecnológica de Colombia, Tunja. Av. Central del Norte. e-mail: saints219@hotmail.com la propagación de romero es ME en proporción 1:1 en volumen. El sustrato ME presentó una mayor eficiencia en el uso del agua (EUA) que el suelo negro de páramo. Una mayor cantidad de agua generó mayor longitud de ramas. La mayor producción de biomasa fresca y seca, se obtuvo con la aplicación del coeficiente de riego de uno y el sustrato ME.

Palabras clave: Invernadero, aromáticas, cascarilla de arroz, eficiencia, evaporación.

\section{SUMMARY}

Little information on irrigation and substrates in nurseries for rosemary propagation under greenhouse conditions, that allows the producer to obtain the total productive capacity of the plant, is available. Under stress conditions the rosemary plant limits the production of dry and fresh biomass. The present work was carriet out with the objective to evaluate the effect of two substrates and different irrigation levels in the propagation of this aromatic plant. An completely random experimental design with ten treatments, product of the combination of five irrigation levels (multiplying coefficients of evapotranspiration $0.6,0.8,1,12$, and 1.4) and two substrates, black soil (BS) and the mixture of BS and burned rice husk $(\mathrm{BRH})$, proportion 1:1, were applied, each treatment with four replica for a total of 40 experimental units (UE). The best coefficient (irrigation levels) for the propagation of cuttings was found to be number one. The best substrate for the propagation of rosemary was the mixture of black soil with rice husk in a 
proportion in volume of $1: 1$. The substrate BRH showed a higher efficiency in water use than the black soil obtained from moor. A higher water availability generated greater branch length. Higher production of fresh and dry biomass was obtained with the application of the coefficient of irrigation number 1 and the substrate $\mathrm{BRH}$.

Key words: Greenhouse, aromatic plants, rice husk, efficiency, evaporation.

\section{INTRODUCCIÓN}

El romero es originario de las regiones del mediterráneo. Los principales productores a escala mundial son Turquía, Marruecos, España, Yugoslavia, Albania, Estados Unidos y México. Colombia, en el 2002, exportó romero a los Estados Unidos, a valores cercanos a los 2,6 US\$ $/ \mathrm{kg}$, con volúmenes de $285 \mathrm{t}$, lo que hace evidente su importancia económica (Proexport, 2002).

El romero soporta situaciones de sequía (Lemes, 2000). No obstante Sánchez-Blanco et al. (2004) probaron tratamientos de estrés hídrico en romero y encontraron que el déficit de agua redujo el crecimiento entre un 40 y $50 \%$, tanto de la parte aérea de la planta como de la altura, el diámetro de tallo y el área foliar. El peso seco total y el potencial del agua de la hoja también disminuyeron con el déficit de riego. Luego de 16 días sin riego, se restableció el suministro de agua a la planta, situación que originó el aumento en el número de estomas.

Westervelt (2003) realizó aplicaciones de agua con diferentes contenidos de humedad en el suelo (20 y $30 \%$ ), encontrando que la lámina de riego no influye en el peso seco de las raíces; no obstante, las láminas de riego más altas presentaron una mayor parte aérea.

Del mismo modo, Westervelt (2003) sometió el suelo a diferentes contenidos de humedad $20 \%$, 30\% y $40 \%$, y obtuvo como resultado que ni la biomasa seca aérea ni de raíz, se vieron afectadas por los diferentes niveles de humedad. La lámina de riego fue significativa para la biomasa seca de raíces y la parte aérea sólo hasta la cuarta semana; las láminas de riego más bajas presentaron las raíces más largas y una mayor parte aérea.

En propagación, Lemes (2000) ensayó tres tipos de esquejes (yemas terminales, intermedias y proximales al tallo) en cinco sustratos y halló que los esquejes de mejor rendimiento fueron los obtenidos a partir de las yemas terminales sembradas en zeolita. El mismo autor también evaluó varias concentraciones y clases de auxinas (ácido indol 3 acético; ácido indol 3 butírico; ácido naftalen-acético; 0, 50 y $100 \mathrm{mg} \cdot \mathrm{kg}^{-1}$ ), encontrando que los esquejes no mostraron respuesta a los reguladores de crecimiento.

Westervelt (2003) evaluó un gran número de sustratos y encontró que los mejores resultados, en cuanto a peso seco de la parte aérea y altura de planta, se presentaron cuando se utilizó turba como sustrato. La turba a pesar de ser un sustrato que en propagación da muy buenos rendimientos disminuye la rentabilidad de los cultivos por su alto costo (Resh, 1997). Por lo tanto, se hace necesario buscar sustratos igual de eficientes pero más económicos, que brinden una adecuada retención de humedad, con el fin de optimizar el uso del recurso hídrico. De acuerdo a lo anterior, el objetivo de este trabajo fue evaluar el efecto de dos sustratos y diferentes láminas de riego en la propagación de romero.

\section{MATERIALES Y MÉTODOS}

El estudio, se realizó en las instalaciones de la Universidad Pedagógica y Tecnológica de Colombia en condiciones de invernadero, a una altitud de $2782 \mathrm{msnm}$ y con las siguientes coordenadas: longitud $73^{\circ} 23^{\prime}$ Oeste, latitud $5^{\circ} 32^{\prime}$ Norte. La temperatura interior del invernadero en promedio fue de $16^{\circ} \mathrm{C}$ y la humedad relativa (H.R.) promedio del $65 \%$.

Como material vegetal, se utilizó esquejes de romero de una longitud de $10 \mathrm{~cm}$, tomados de la parte apical de la planta, los cuales se sembraron en materas, con una capacidad de $3000 \mathrm{~cm}^{3}$, aproximadamente, para $2 \mathrm{~kg}$ de sustrato. Así mismo, se utilizaron dos sustratos: el primero fue suelo negro proveniente de páramo (SN) y, el otro una mezcla de suelo negro y cascarilla de arroz quemada, en proporción 1:1 en volumen (ME) (Tabla 1).

Se empleó un diseño experimental completamente al azar con diez tratamientos, los cuales fueron la combinación de cinco láminas de riego, coeficientes multiplicadores de la evapotranspiración 0,6; 0,8; 1; 1,2; y 1,4 y dos sustratos ( $\mathrm{SN}$ y $\mathrm{ME}$ ); cada tratamiento tuvo cuatro repeticiones para un total de cuarenta unidades experimentales (UE). 
Tabla 1. Propiedades fisicoquímicas de los sustratos utilizados en la propagación de romero.

\begin{tabular}{|l|c|c|c|}
\hline \multicolumn{1}{|c|}{ Propiedad } & Suelo negro & Cascarilla de arroz quemada & Unidades \\
\hline Densidad Real & 2,3 & - & $\mathrm{g} / \mathrm{cm}^{3}$ \\
\hline Densidad Aparente & 1,2 & 0,16 & $\mathrm{~g} / \mathrm{cm}^{3}$ \\
\hline Porosidad & 48 & $80-90$ & $\%$ \\
\hline Og(cc) & $30-40$ & $45-55$ & $\%$ \\
\hline $\mathrm{pH}$ & 4,8 & 6 & Escala \\
\hline $\mathrm{M} . \mathrm{O}$. & 4,80 & - & $\%$ \\
\hline $\mathrm{CA}$ & 1,58 & 0,024 & $\mathrm{cmol} / \mathrm{kg}$ de suelo \\
\hline $\mathrm{Mg}$ & 0,27 & 0,018 & $\mathrm{cmol} / \mathrm{kg}$ de suelo \\
\hline $\mathrm{K}$ & 0,16 & 0,0039 & $\mathrm{cmol} / \mathrm{kg}$ de suelo \\
\hline $\mathrm{Na}$ & 0,88 & - & $\mathrm{cmol} / \mathrm{kg}$ de suelo \\
\hline $\mathrm{P}$ & 9,25 & 0,08 & $\mathrm{mg} / \mathrm{kg}$ \\
\hline
\end{tabular}

La evapotranspiración (Ept), se midió con dos evaporímetros plásticos a escala del tanque de evaporación clase A, de dimensiones $29 \mathrm{~cm}$ de diámetro por $6,1 \mathrm{~cm}$ de profundidad. Éstos, se instalaron dentro del invernadero, con el fin de tener un dato más exacto. Para el suministro de agua a la planta, se utilizó un sistema de riego por goteo. Las aplicaciones, se realizaron con base en la siguiente fórmula:

$$
\text { Lámina }=\frac{\operatorname{Etp}^{*} C^{*} A}{\eta_{r}}
$$

En donde, Etp = evapotranspiración en mm medida en el tanque evaporímetro; $\mathrm{C}=$ coeficiente multiplicador; $\mathrm{A}=$ área de la matera $\left(254,4 \mathrm{~cm}^{2}\right) ; \eta_{\mathrm{r}}=$ eficiencia del riego por goteo $(0,9)$.

Las variables de respuesta medidas al cabo de cuatro meses fueron: altura de planta; biomasa fresca y seca de tallo, de hojas, de raíces y total; longitud promedio de ramas por planta; eficiencia agronómica en el uso del agua (EUA) calculada dividiendo la biomasa seca en la cantidad de agua aplicada por tratamiento, acorde a lo realizado por Wu et al. (2008); porcentaje de esquejes enraizados y contenido relativo de agua (CRA), siguiendo la metodología propuesta por Weatherley $\mathcal{E}$ Barrs (1965). Al final del experimento, se midieron estos últimos parámetros, pues este es el momento en el que las plantas se llevan a campo, lo que permite conocer el vigor del material que va a ser trasplantado.
Se realizaron análisis de varianza (Anova), para determinar la existencia de diferencias entre tratamientos. Así mismo, mediante la prueba de comparación de Tukey al $5 \%$ se establecieron los mejores tratamientos. Como los datos no se ajustaron a una distribución normal por el número elevado de plantas que murieron, debido al efecto negativo de algunos coeficientes, se procedió a la realización de un Anova no paramétrico. El software SAS v. 8.1e, se utilizó para realizar las pruebas estadísticas.

\section{RESULTADOS Y DISCUSIÓN}

Altura de planta: Esta variable presentó diferencias significativas entre coeficientes de riego y la prueba de Tukey indica que el coeficiente de mejor comportamiento en cuanto altura de las plantas es el de 1; también muestra que entre los coeficientes de 1,$4 ; 0,8$ y 1,2 no hay diferencias, pero al analizar el coeficiente de 0,6 éste muestra diferencias con el resto de los coeficientes, siendo el de menor respuesta (Figura 1). Estos resultados reflejan que el romero, en su etapa de propagación, mantiene unos niveles medios en cuanto a consumo de agua, es decir, que la planta en este estado no requiere grandes suministros de agua para su crecimiento, ni tampoco soporta un déficit hídrico prolongado, pues en las dos situaciones disminuye la respuesta fisiológica de crecimiento.

Por lo tanto, con restituir el agua que se pierde tanto por evaporación como por transpiración es suficiente 


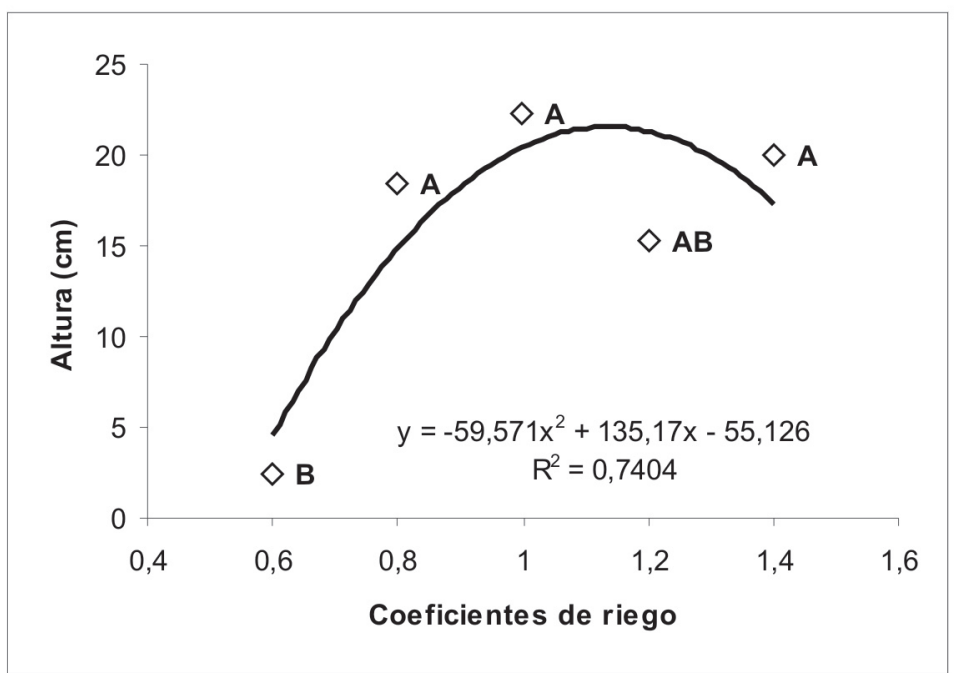

Figura 1. Altura de plantas de romero en propagación sometidas a diferentes láminas de riego y sustratos. Promedios de cuatro repeticiones. Letras distintas indican diferencias significativas de acuerdo con la prueba de Tukey $(\alpha=0.05)$.

para el desarrollo de la planta, situación que concuerda con lo reportado por Sánchez-Blanco et al. (2004), quienes afirman que los tratamientos con déficit hídrico originaron una disminución en la altura. Esto se da porque con bajos niveles de agua la presión de turgor de la célula no es tan alta y la pared celular no se ve obligada a expandirse (Marschner, 2002), lo que resulta en una altura reducida.

En cuanto a los sustratos existen diferencias significativas con respecto a la variable altura. El sustrato que mejor se comportó fue ME en proporción 1:1, muy superior al sustrato SN (Figura 2). Esta situación se pudo dar debido a que el suelo negro posee una menor porosidad que cuando esta mezclado con cascarilla de arroz (Tabla 1), lo que hace que se presente una mayor facilidad para la penetración inicial de las raíces a través del sustrato y mayor disponibilidad de oxígeno (Traní et al. 2007), con lo cual, la planta adquiere vigor más rápidamente en el sustrato ME. Así mismo, el sustrato ME presenta un mayor contenido de humedad gravimétrico $(\theta g)$ (Tabla 1 ), indicando que las plantas al disponer de una mayor cantidad de agua tienen una mayor altura, debido a que el gasto energético que la planta emplea para su crecimiento disminuye cuando existe agua aprovechable en el suelo (Montenegro \& Malagón, 1990).

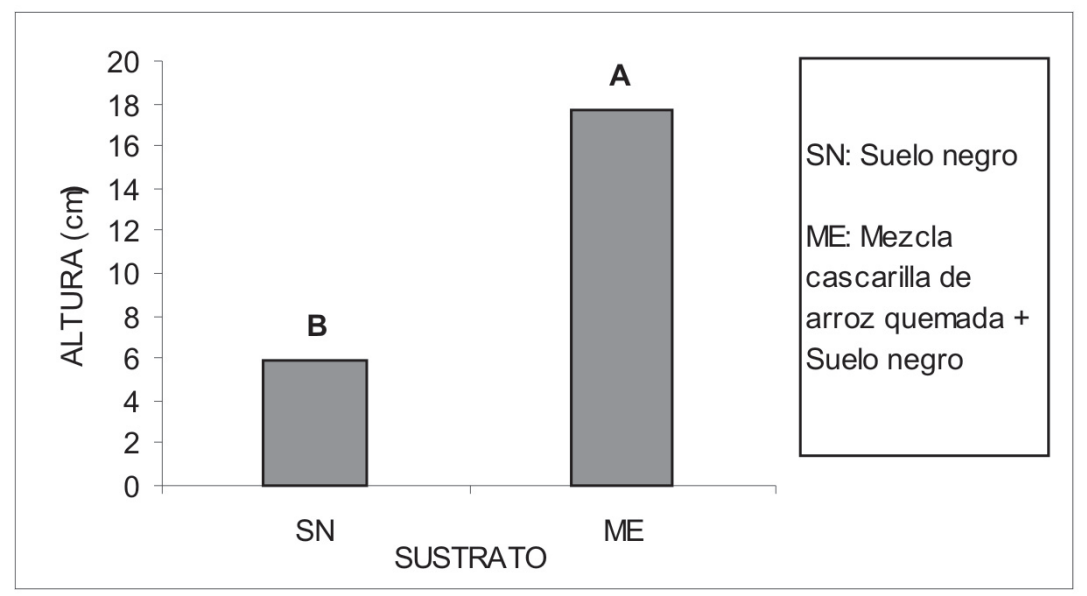

Figura 2. Altura de plantas de romero en propagación bajo dos sustratos. Promedios de cuatro repeticiones. Letras distintas indican diferencias significativas, de acuerdo con la prueba de Tukey $(\alpha=0,05)$. 
Al analizar los resultados obtenidos es claro que la propagación del romero requiere de sustratos adecuados, que permitan facilidad de penetración y de expansión de raíces, retención de humedad y unos niveles adecuados de aireación, que brinden un acceso al agua con bajo gasto en cuanto niveles de energía por parte de la planta para el buen desarrollo del esqueje; ésto, con el fin de mejorar el vigor y la altura de la planta. Paulus et al. (2005) evaluaron diferentes sustratos y encontraron que la mejor respuesta en la altura de la menta se dio cuando utilizaron un sustrato compuesto por la mezcla de materiales orgánicos y minerales y las menores respuestas se presentaron cuando se evaluaron los sustratos por separado.

En este estudio, con la mezcla se logra mejores resultados pues combina el sustrato SN con buenas propiedades químicas, con un sustrato de cascarilla de arroz quemada, que presenta propiedades físicas excelentes, lo que hace la mezcla ME un medio óptimo para la propagación.
Biomasa fresca y seca de tallo, hojas, raíz y total: Estas variables se vieron afectadas solo por el sustrato, pues el coeficiente de riego no mostró diferencias significativas, lo que indica que las cantidades de agua aplicadas no influyeron directamente en la propagación de romero. Para las variables de masa fresca y seca de tallo, hojas, raíz y total, se presentaron diferencias significativas. El sustrato que presentó el mejor comportamiento fue el $\mathrm{ME}$, con un promedio superior al arrojado por el sustrato SN (Figura 3).

El coeficiente de riego probablemente no influyó en la biomasa fresca y seca de raíces, de tallos y de hojas, debido a que en este caso, el proceso de propagación esta determinado principalmente por el sustrato, pues la aplicación de cantidades elevadas de agua en sustratos con baja retención de humedad no tienen efecto en la cantidad de agua aprovechable que el sustrato pueda brindar a la planta, la cual esta determinada por las propiedades físicas del sustrato (Lal \& Shukla, 2004). Una breve mirada indicaría que

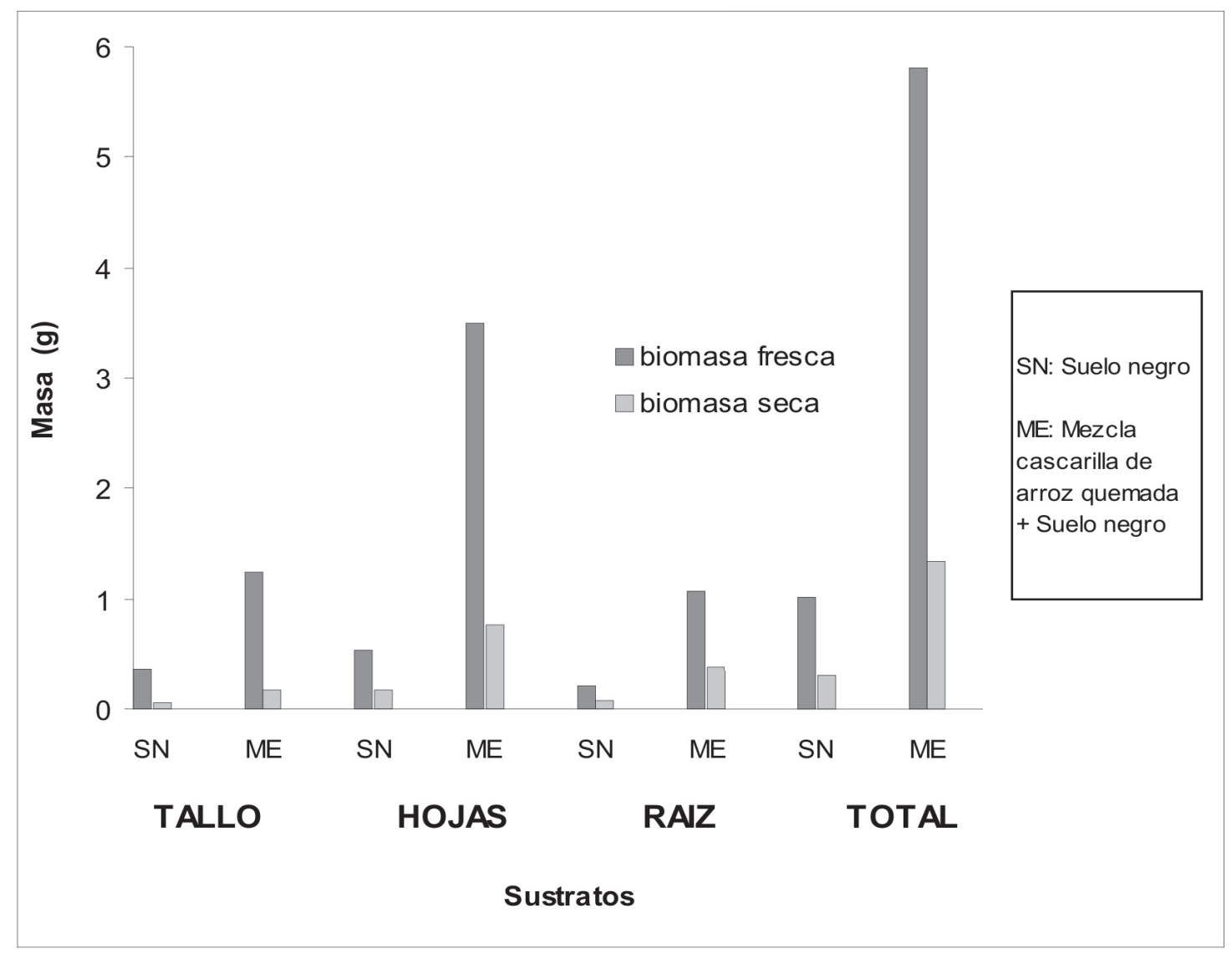

Figura 3. Biomasa fresca y seca de tallo, hojas, raíz y total para romero en propagación en dos sustratos. 
la productividad de la planta depende del sustrato, de acuerdo a sus características físicas, hídricas, químicas y biológicas y de sus interacciones (Francis et al. 2005).

El tipo de sustrato ME ofrece condiciones adecuadas para la propagación de romero, ya que favorece el crecimiento de las raíces y, éste, a su vez, aumenta la absorción de agua y de nutrientes que promueven la síntesis de compuestos fotoasimilados en la parte aérea, razón por la cual se registra el incremento de peso fresco y seco en las estructuras de la planta.

Del mismo modo restricciones químicas, como el exceso de aluminio en el suelo de páramo, el cual incrementa la acidez (Espinosa, 2001) (Tabla 1), se ven disminuidas al mezclar el SN con cascarilla de arroz quemada, el cual es un sustrato inerte (Resh, 1997), situación que genera una mayor disponibilidad de nutrientes en el sustrato $\mathrm{ME}$, ya que el $\mathrm{pH}$ de la mezcla es cercano a la neutralidad.

La mayor biomasa de raíces tuvo un valor promedio de $0,46 \mathrm{~g}$ y se presentó en el coeficiente de 1 , comparada con los demás coeficientes $(0,6 ; 0,8 ; 1,2 ;$ y 1,4$)$, los cuales presentaron 0,$09 ; 0,29 ; 0,16$ y 0,28 , respectivamente. A pesar de no encontrar diferencias significativas, el crecimiento de las raíces con altos niveles de agua disminuye, debido a una menor difusión de oxígeno en la zona radicular (Xie et al. 2008), así como también el déficit hídrico, pues la falta de agua disminuye el crecimiento de la raíz (Mainiero \& Kazda, 2004).
Contenido relativo de agua (CRA): Para esta variable, se evidenciaron diferencias significativas entre tratamientos. El de mejor promedio fue el correspondiente al coeficiente de 1 , seguido por el de 1,4 y, éste, a su vez, seguido por 0,$8 ; 1,2 ; 0,6$, lo que indica que el coeficiente que expresó el mayor CRA es el equivalente al de suministrar la misma cantidad de agua evapotranspirada (Figura 4). El CRA determina el contenido hídrico que tiene la planta en relación con el que tendría la planta si estuviera muy bien hidratada (Salisbury \& Ross, 1994). Es claro que la lámina correspondiente al coeficiente de 1 brinda una mejor relación hídrica para la planta de romero mostrando unos niveles de estrés hídrico menores, por lo que ésta será fotosintéticamente eficiente y durante más tiempo, logrando un mayor incremento de la masa vegetal.

El sustrato ME fue superior con respecto al SN, pero no lo suficiente para presentar diferencias significativas, por lo que se deduce que el sustrato no influye en el CRA de las plántulas de romero, si no que el suplemento hídrico en los últimos días al corte y las condiciones ambientales de humedad y de temperatura presentes a la hora de la realización de los muestreos afectan el contenido de agua en las hojas (Kaiser, 1987; MunnéBosh et al. 2000).

Como ocurrió con el peso seco y fresco, los excesos de agua no incrementan el agua disponible para la planta en el sustrato, además tanto el sustrato $\mathrm{SN}$ como el ME poseen $\theta_{g}$ similares (Tabla 1 ), que minimizan el efecto del sustrato en el CRA.

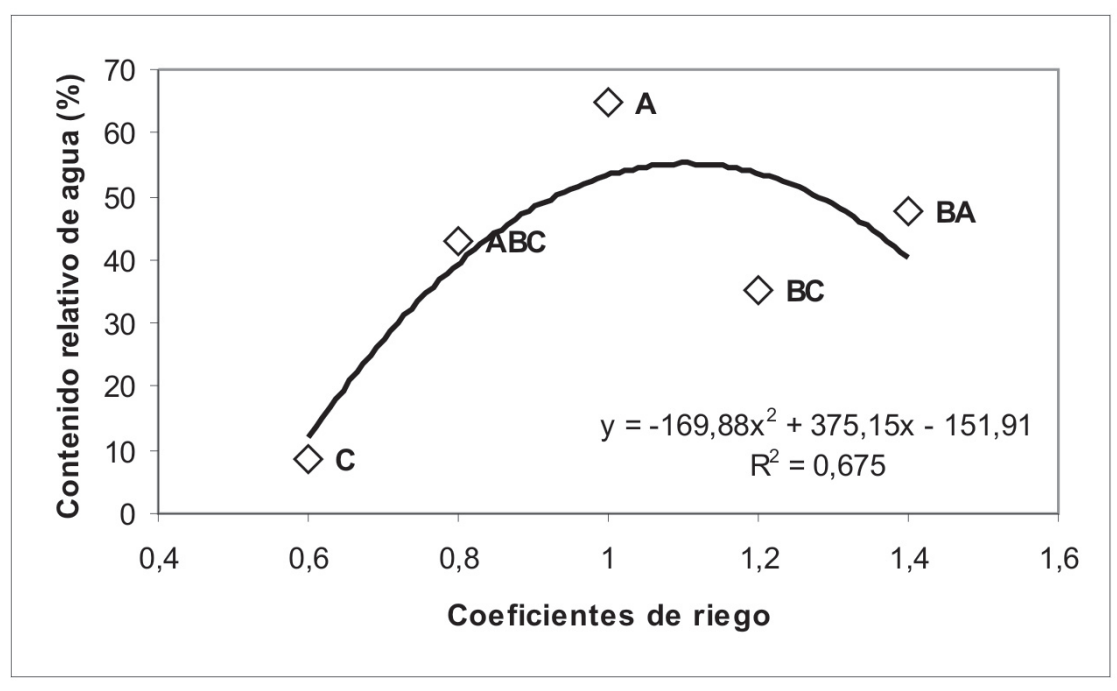

Figura 4. Contenido relativo de agua de plantas de romero bajo diferentes láminas de riego. Promedios de cuatro repeticiones. Letras distintas indican diferencias significativas de acuerdo con la prueba de Tukey $(\alpha=0,05)$. 
Los valores de CRA para los coeficientes más altos $(0,8$; 1 y 1,4$)$ presentaron valores que oscilan entre el $40 \%$ y el $65 \%$ y las plántulas en ningún momento mostraron síntomas de déficit hídrico, contrario a Munné-Bosch et al. (2000), quienes determinaron que en las hojas de romero el CRA de agua disminuye en el verano y que valores por debajo del $70 \%$ comienzan a causar estrés hídrico en las plantas, mientras que CRA por debajo del $30 \%$ originan daños irreversibles. También encontraron que, debido a las diferencias en precipitación, el CRA varió entre un $42 \%$ y un $80 \%$. Bajo condiciones de invernadero, el follaje generalmente no se humedece, por lo que el CRA tiende a ser constante y de menor valor, contrario a lo que sucede a campo abierto. El coeficiente de 1,2 presenta valores bajos en todas las variables, debido a que presentó problemas de prendimiento que retrasaron el crecimiento y repercutieron al final.

Longitud promedio de ramas por plántula: Se hallaron diferencias significativas entre coeficientes de riego de acuerdo a la longitud promedio de ramas por plántula. El tratamiento correspondiente al coeficiente de 1,4 obtuvo un promedio de $1,89 \mathrm{~cm}$, siendo superior al presentado por los coeficientes de $1 ; 0,8 ; 1,2$ y 0,6, los cuales presentaron promedios de 1,$60 ; 1,11 ; 0,36$ y 0 $\mathrm{cm}$, respectivamente (Figura 5).

Si se tiene en cuenta que el crecimiento está relacionado con la presión de turgencia y con las relaciones hídricas, el crecimiento de una célula, entonces, depende de las características de su pared celular, de la membrana plasmática y de la presión de turgencia, así cualquier descenso en la presión de turgencia se traducirá en una disminución en el crecimiento del volumen celular (Salisbury \& Ross, 1994), por lo que una mayor lámina de agua aplicada favorecerá el proceso de elongación celular y, a su vez, la longitud de las ramas.

El crecimiento en volumen es el parámetro fisiológico que más rápidamente se afecta por un déficit hídrico, razón por la cual desciende la síntesis proteica, es decir, disminuyen las enzimas, como la nitrato reductasa, pero se da un aumento del ácido abscísico (ABA) que actúa en la apertura y cierre de los estomas. De esta forma, la planta ahorrará más agua, debido a la disminución en la apertura estomática, por lo que tendrá dificultad para captar el $\mathrm{CO}_{2}$ y disminuirá la fotosíntesis (Marschner, 2002; Salisbury \& Roos, 1994).

Se puede decir que en la planta los coeficientes de riego de 1, 0,8, 1,2 y 0,6, generan mayor estrés hídrico que el coeficiente de 1,4, el cual, al poseer un mayor aporte de agua, favorece una mayor longitud de sus ramas.

Para el sustrato no se encontraron diferencias significativas. Se considera que éste no influye significativamente sobre la longitud promedio de ramas. El hecho de que en la propagación de romero se hayan

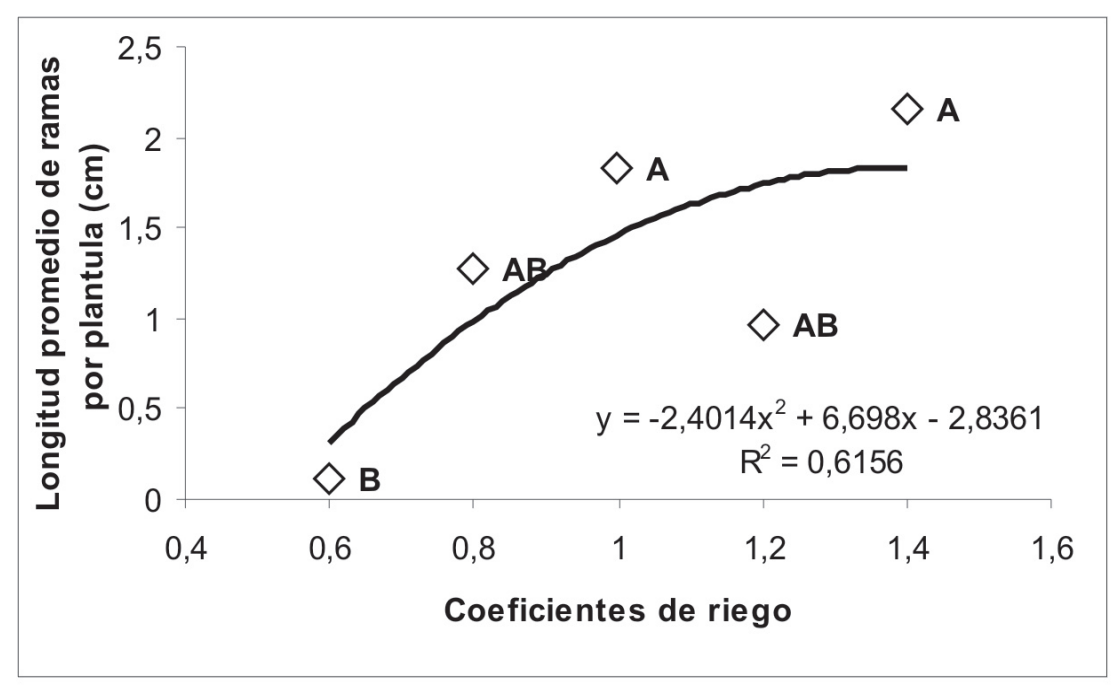

Figura 5. Longitud promedio de ramas por plántula de romero en propagación bajo diferentes láminas de riego. Promedios de cuatro repeticiones. Letras distintas indican diferencias significativas de acuerdo con la prueba de Tukey $(\alpha=0,05)$. 
encontrado diferencias significativas entre la longitud de ramas y no entre pesos frescos para diferentes láminas de riego, implica que las ramas provenientes de los tratamientos, con mayor aplicación de agua, son más largas, pero poseen menor densidad, lo que las hace de igual peso que a las ramas más cortas.

Eficiencia en el uso del agua: No se presentaron diferencias significativas entre coeficientes de riego respecto a la eficiencia del consumo de agua de los tratamientos. Los sustratos afectaron significativamente la eficiencia en el uso del agua. El sustrato que mejor se comportó fue el $\mathrm{ME}$, con un promedio de $0,10 \mathrm{~g} / \mathrm{kg}$ de agua aplicada, siendo superior al presentado por el sustrato SN con un promedio de 0,02 $\mathrm{g}$ de biomasa seca por $\mathrm{kg}$ de agua aplicada. Estas eficiencias resultan ser adecuadas para el sustrato ME al compararlas con las presentadas por Davidoff $\mathcal{E}$ Hanks (1989) quienes obtuvieron una eficiencia de $0,4 \mathrm{~g} / \mathrm{kg}$, lo que indica que para la producción de un $\mathrm{kg}$ de biomasa seca se transpiran $230 \mathrm{~kg}$ de agua, sin tener en cuenta la evaporación. Para el sustrato SN la eficiencia es muy baja lo que expresa que por cada kg de biomasa seca se deben aplicar 5000kg de agua. No obstante, los valores de EUA presentados por las plántulas de romero resultan bajos si se comparan con los encontrados por Wu et al. (2008) para Sophora davidii, los cuales muestran valores de entre 0,4 y $1,2 \mathrm{~g} / \mathrm{kg}$.

Trubat et al. (2008) afirman que el incremento en la EUA se da como mecanismo de defensa a condiciones de sequía. Sin embargo, Wu et al. (2008) encontraron que en $S$. davidii la EUA disminuyó con el aumento del nivel de estrés hídrico, debido a una menor cantidad en la producción de biomasa. Así mismo, afirman que la EUA correlaciona positivamente con el área foliar, la biomasa y la longitud de raíces y mencionan que esta última determina la EUA, pues a mayor longitud de raíces la absorción de agua y nutrientes es mayor.

Porcentaje de esquejes enraizados: Se encontró que las láminas que mejor comportamiento tuvieron en el porcentaje de enraizamiento de los esquejes fueron las de los coeficientes de 1 y 1,4 , con un $75 \%$. El sustrato que mejor se comportó fue el $\mathrm{ME}$, con un promedio general de enraizamiento del 55\%, muy superior al presentado por el sustrato SN, el cual registró un promedio del $35 \%$. Lo anterior es posible debido a que la mezcla presenta una mayor aireación y, a su vez, una buena retención de humedad que favorece la emisión de raicillas en la etapa de propagación.
Los modelos matemáticos obtenidos para las variables altura, CRA y longitud promedio de ramas por plántula presentan coeficientes de correlación entre 0,6 y 0,8, y se ajustan al tipo polinomial. El tratamiento del coeficiente 1,2 presentó dificultades de prendimiento que afectaron el desarrollo del mismo y la obtención de un modelo más ajustado. En general, el valor de coeficiente de riego óptimo oscila entre 1,1 y 1,2 para la altura de plantas y el CRA, mientras que para la longitud promedio de ramas por plántula es de 1,4.

\section{CONCLUSIONES}

Una mayor cantidad de agua genera una mayor longitud de ramas en la propagación de romero.

La mezcla de suelo negro de páramo más cascarilla de arroz en proporción 1:1 en volumen presentó mayor eficiencia en el uso del agua, altura de plántula y biomasa fresca y seca de raíces, tallo y hojas.

La mayor producción de biomasa fresca y seca, se obtuvo con la aplicación del coeficiente de riego de 1 y el sustrato ME.

La máxima altura y CRA, se presentan con los coeficientes de 1,1 y 1,2 , respectivamente y para la longitud promedio de ramas por plántula, en 1,4.

\section{BIBLIOGRAFÍA}

DAVIDOFF, B.; HANKS R. J. 1989. Sugar beet production as influenced by limited irrigation. Irrigation Science 10:1-17.

ESPINOSA, J. 2001. Acidez y encalado de los suelos. En: Silva, F. (ed). Fertilidad de Suelos. Diagnóstico y control. $2^{a}$ edición. Sociedad Colombiana de la Ciencia del Suelo. p.113-128.

FRANCIS, R.A.; GURNELL, A.M.; PETTS, G.E.; EDWARDS, P.J. 2005. Survival and growth responses of Populus nigra, Salix elaeagnos and Alnus incana to varying levels of hydric stress. Forest. Ecol. Managem. 210:291-301.

KAISER, W.M. 1987. Effects of water deficit on photosynthetic capacity. Physiol. Plant. 71:142-149. 
LAL, R.; SHUKLA, M.K. 2004. Principles of Soil Physics. Marcel Dekker, New York. 716p.

LEMES, H. 2000. Multiplicación vegetativa de Rosmarinus officinalis L. Romero. Rev. Cubana Plant Med. 3:79-82.

MAINIERO, R.; KAZDA, M. 2004. Effects of Carex restrata on soil oxygen in relation to soil moisture. Plant Soil 270:311-320.

MARSCHNER, H. 2002. Mineral nutrition of higher plants. 2a ed. Editorial Academis Press London. 889p.

MONTENEGRO, H.; MALAGÓN, D. 1990. Propiedades físicas de los suelos. Bogotá. IGAC. 813p.

MUNNÉ-BOSCH, S.; ALEGRE, L.; SCHWARZ, K. 2000. The formation of phenolic diterpenes in Rosmarinus officinalis L. under mediterranean climate. Eur. Food Res. Technol. 210:263-267.

PAULUS, D.; MEDEIROS, S.L.P.; SANTOS, O.S; RIFFEL, C.; FABBRIN, G.; PAULUS, E. 2005. Substratos na produção hidropônica de mudas de hortelã. Hort. Bras. 23(1):48-50.

PROEXPORT. 2002. Exportaciones colombianas totales según sectores de promoción 2002. Disponible desde Internet en: http://www.proexport.com.co/intelexport/ aplicacion/frames.asp. (con acceso 15/05/03).

RESH, H.M. 1997. Cultivos hidropónicos: Nuevas técnicas de producción. $4^{a}$ ed. Ed. Mundi-Prensa. Madrid. 509p.

SALISBURY, F.; ROSS, C. 1994. Fisiología vegetal. Madrid: Ibero América. 759p.
SÁNCHEZ-BLANCO, M.J.; FERNÁNDEZ, T.; NAVARRO, A.; BAÑON, S.; ALARCÓN, J. 2004. Effects of irrigation and air humidity preconditioning on water relations, growth and survival of Rosmarinus officinalis plants during and after transplanting. J. Plant Physiol. 161(10):1133-1142.

TRANI, P.; FELTRIN, D.; POTT, C.; SCHWINGEL, M. 2007. Avaliação de substratos para produção de mudas de alface. Hort. Bras. 25(2):256-260.

TRUBAT, R.; CORTINA, J.; VILAGROSA, A. 2008. Short-term nitrogen deprivation increases field performance in nursery seedlings of Mediterranean woody species. Environ. J. Arid Environments 72:879-890.

WEATHERLEY, P.E.; BARRS, C. 1965. A re-examination of the relative turgidity technique for estimating water deficit in leaves. Austral. J. Plant. Physiol. 15:413-428.

WESTERVELT, P. 2003. Greenhouse production of Rosmarinus officinalis L. Thesis Faculty Virginia Polytechnic Institute and State University. Master of Science in Horticultural Science, Blacksburg, Virginia. 51p.

WU, F.; BAO, W.; LI, F.; WU, N. 2008. Effects of drought stress and $N$ supply on the growth, biomass partitioning and water-use efficiency of Sophora davidii seedlings. Environ. Exp. Bot. 63:248-255.

XIE, Y.; LUO, W.; WANG, K.; REN, B. 2008. Root growth dynamics of Deyeuxia angustifolia seedlings in response to water level. Aquatic Bot. En prensa. 5p.

Recibido: Septiembre 21 de 2007

Aceptado: Mayo 7 de 2008 